\title{
Access-site complications in ultrasound-guided endovascular thrombectomy: a single-institution retrospective cohort study
}

\author{
Connor T. A. Brenna, BSc, ${ }^{1}$ Jerry C. Ku, MD, ${ }^{2,3}$ Christopher R. Pasarikovski, MD, ${ }^{2,3}$ \\ Stefano M. Priola, MD, ${ }^{4}$ Erin E. Dyer, MD, ${ }^{5}$ Peter Howard, MD, ${ }^{6}$ Ashish Kumar, MBBS, $M C^{2},{ }^{2,3}$ \\ Leodante da Costa, MD, MSc, ${ }^{2,3,6}$ and Victor X. D. Yang, MD, PhD ${ }^{2,3}$
}

1Department of Medicine and 'Division of Neurosurgery, Department of Surgery, University of Toronto; ${ }^{3}$ Division of Neurosurgery, Sunnybrook Health Sciences Centre, Toronto; ${ }^{4}$ Department of Neurosurgery, Health Sciences North, Northern Ontario School of Medicine, Sudbury; ${ }^{5}$ Division of Neurosurgery, Windsor Regional Hospital, Windsor; and ${ }^{6}$ Division of Neuroradiology, Department of Medical Imaging, Sunnybrook Health Sciences Centre, Toronto, Ontario, Canada

\begin{abstract}
OBJECTIVE Mechanical endovascular thrombectomy (EVT) is an increasingly relied-on treatment for clot retrieval in the context of ischemic strokes, which otherwise are associated with significant morbidity and mortality. Despite several known risks associated with this procedure, there is a high degree of technical heterogeneity across both centers and operators. The most common procedural complications occur at the point of transfemoral access (the common femoral artery), and include access-site hematomas, dissections, and pseudoaneurysms. Other interventional fields have previously popularized the use of ultrasound to enhance the anatomical localization of structures relevant to vascular access and thereby reducing access-site complications. In this study, the authors aimed to describe the ultrasound-guided EVT technique performed at a large, quaternary neurovascular referral center, and to characterize the effects of ultrasound guidance on access-site complications.
\end{abstract}

METHODS A retrospective chart review of all patients treated with EVT at a single center between January 2013 and August 2020 was performed. Patients in this cohort were treated using a universal, unique, ultrasound-guided, singlewall puncture technique, which bears several theoretical advantages over the standard technique of arterial puncture via palpation.

RESULTS There were 479 patients treated with EVT within the study period. Twenty patients in the cohort were identified as having experienced some form of access-site complication. Eight (1.67\%) of these patients experienced minor access-site complications, all of which were groin hematomas and none of which were clinically significant, as defined by requiring surgical or interventional management or transfusion. The remaining 12 patients experienced arterial dissection $(n=5)$, arterial pseudoaneurysm $(n=4)$, retroperitoneal hematoma $(n=2)$, or arterial occlusion $(n=1)$, with only $1.04 \%$ $(5 / 479)$ requiring surgical or interventional management or transfusion.

CONCLUSIONS The authors found an overall reduction in total access-site complications as well as minor access-site complications in the study cohort compared with previously published randomized controlled trials and observational studies in the recent literature. The findings suggested that there may be a role for routine use of ultrasound-guided puncture techniques in EVT to decrease rates of complications.

https://thejns.org/doi/abs/10.3171/2021.4.FOCUS2198

KEYWORDS endovascular thrombectomy; ultrasound; access-site complications; transfemoral puncture; ischemic stroke

$\mathrm{M}$ ECHANICAL endovascular thrombectomy (EVT) has emerged as the standard of care for the treatment of ischemic strokes in select patients up to 6 to 24 hours from symptom onset. ${ }^{1,2}$ The proportion of patients with ischemic stroke who received EVT increased sixfold between 2004 and 2009. ${ }^{3}$ However, the widespread pursuit of greater recanalization rates has led to considerable heterogeneity in its technique. A survey published by

ABBREVIATIONS CFA = common femoral artery; EVT = endovascular thrombectomy; $\mathrm{MCA}=$ middle cerebral artery; $\mathrm{RCT}=$ randomized controlled trial; tPA = tissue plasminogen activator; US = ultrasound; VCD = vascular closure device.

SUBMITTED February 18, 2021. ACCEPTED April 6, 2021.

INCLUDE WHEN CITING DOI: 10.3171/2021.4.FOCUS2198. 
Mehta and colleagues in 2019 identified dozens of technical variations in EVT, ${ }^{4}$ highlighting the need for evidencebased best-practice guidelines. EVT is widely performed with transfemoral vascular access, with ultrasonography as a useful adjunct to the standard technique of palpationguided puncture. ${ }^{5}$ Transradial access is also emerging as an alternative route of access for neuroendovascular procedures, including EVT. ${ }^{6-8}$ In rare cases, direct punctures of the carotid ${ }^{9,10}$ or vertebral ${ }^{11,12}$ arteries have also been utilized to circumvent the challenges of tortuous vascularity in select patients (at the cost of certain unique procedural risks such as neck hematoma).

Access-site complications account for the most common adverse events across all peripheral vascular interventions (occurring in $1 \%-11 \%$ of these procedures). ${ }^{13}$ For EVT, access-site complications have been reported to occur in between $2.78 \%$ and $5.13 \%$ of cases ${ }^{14,15}$ and represent a common and actionable target for improvement in all interventions requiring transfemoral access. Outside of interventional neuroradiology, the use of ultrasound (US) to guide femoral access has garnered attention as one possible means of curtailing these complications. A 2019 systematic review and meta-analysis by Sorenson and colleagues compared US-guided and non-US-guided femoral access and found that US conferred a superior time-to-access (24.90 seconds), first-pass success rate (OR 2.97), and lower access-site complication rate (OR 0.42). ${ }^{5}$ US guidance has been ascribed theoretical utility in appropriate anatomical landmarking and sheath placement, and there are compelling reasons to believe that its use may confer a reduction in the access-site complications associated with transfemoral access EVT..$^{16,17}$

For neuroendovascular procedures requiring transfemoral access at our institution, we practice the routine use of US-guided access. The objectives of this retrospective review were to describe a US-guided EVT technique at our large, quaternary neurovascular referral center ${ }^{18}$ and to characterize the effect of US guidance on access-site complications in transfemoral punctures with large, $8 \mathrm{~F}$ catheter sheaths. We hypothesized that US guidance in EVT would reduce groin complication rates associated with transfemoral access compared with rates reported in the literature.

\section{Methods \\ Study Design}

The electronic medical records of all patients treated with EVT at Sunnybrook Health Sciences Centre (Toronto, ON, Canada) between January 2013 and August 2020 were retrospectively reviewed. Of the 479 patients included in this cohort, 216 had been previously characterized as part of a broader analysis of interventional neuroradiology complications in 2019. ${ }^{19}$ All patients who received EVT with transfemoral access for large-vessel occlusion were included in the study, identified through an institutional interventional radiology database of EVT procedures performed within the eligible time window. Exclusion criteria included incomplete documentation of procedural complications, transfemoral access for neuroendovascular procedures other than EVT, non-transfemoral access, and less than 2 weeks of follow-up. Demographic information, clinical presentation, procedural details, and outcomes were collected for each study patient.

To allow for direct comparison with previous reports of groin complications in EVT, we applied the same categorization schema for access-site complication severity used by Oneissi and colleagues..$^{14}$ Specifically, minor complications were defined as groin hematomas and groin hemorrhages, while major complications included retroperitoneal hematomas, arteriovenous fistulae, artery pseudoaneurysms, artery dissections, nerve injuries, arterial occlusions, and wound infections.

Two researchers independently reviewed the records of all patients in the study meeting criteria for either minor or major access-site complications to limit any potential sources of bias. Statistical analysis was limited to descriptive statistics. This study received ethics approval by the Sunnybrook Health Sciences Centre institutional research ethics board. We followed the STROBE guidelines for reporting observational cohort studies. ${ }^{20}$

\section{EVT Technique}

The use of US guidance is routine at our institution for transfemoral access. Patients undergoing EVT are provided conscious sedation, then prepared and draped in a sterile fashion. Both US and palpation are used to localize the common femoral artery (CFA), paying special attention to remain above the bifurcation of the superficial femoral artery and below the inguinal ligament. Single-wall puncture of the CFA is performed under US visualization, and an $8 \mathrm{~F}$ arterial sheath is placed and attached to a continuous heparinized flush. The treating neurointerventionalist selects a 6F catheter (either Penumbra Neuron MAX 088 or Stryker FlowGate BGC), and a roadmapping technique is used to navigate the catheter to a site distal to the occlusion. Angiography is performed to further characterize the occlusion in real time, and a microguidewire is advanced through the catheter to the site of the clot. A reperfusion catheter is advanced coaxially over the microguidewire, and the microguidewire is removed. The reperfusion catheter is attached to an aspiration pump (Penumbra or Stryker), which is activated to engage the clot. The reperfusion catheter is withdrawn coaxially through a $6 \mathrm{~F}$ catheter, which is then used to repeat angiography for assessment of the former occlusion or distal emboli. In a minority of cases, intraarterial tissue plasminogen activator (tPA) is then injected through the $6 \mathrm{~F}$ catheter to assist with the breakdown of any remaining clots.

After the procedure is terminated, catheters are extracted through the groin sheath, and a femoral artery run is performed to confirm puncture site suitability for vascular closure device (VCD) use. In the majority of cases, an 8F Angio-Seal (Terumo Medical Corp.) device is applied for hemostasis; in a minority, when repeat partial thromboplastin time is within a normal range or with puncture sites unsuitable for VCD due to the presence of dissection or proximity to branching vessels despite US guidance, manual compression is used instead. Patients are transferred to the postanesthesia care unit, undergo brain CT within 24 hours, and receive routine monitoring for postprocedural complications, including those at the transfemoral access site. 
TABLE 1. Descriptive analysis of patients

\begin{tabular}{|c|c|}
\hline Characteristic & Value \\
\hline No. of patients & 479 \\
\hline \multicolumn{2}{|l|}{ Sex } \\
\hline$M$ & $237(49)$ \\
\hline $\mathrm{F}$ & $242(51)$ \\
\hline \multicolumn{2}{|l|}{ Age, yrs } \\
\hline Mean \pm SD (range) & $71 \pm 14.5(19-99)$ \\
\hline \multicolumn{2}{|l|}{ Age group } \\
\hline $0-40$ & $14(3)$ \\
\hline $40-60$ & $86(18)$ \\
\hline $60-80$ & $236(49)$ \\
\hline $80+$ & $143(30)$ \\
\hline \multicolumn{2}{|l|}{ Thrombus location } \\
\hline \multicolumn{2}{|l|}{ Side (if lateralized) } \\
\hline $\mathrm{Lt}$ & $223(47)$ \\
\hline Rt & $198(41)$ \\
\hline \multicolumn{2}{|l|}{ Territory } \\
\hline MCA & $343(72)$ \\
\hline ICA & $71(15)$ \\
\hline Basilar & $56(12)$ \\
\hline PCA & $2(0)$ \\
\hline CCA & $1(0)$ \\
\hline VA & $1(0)$ \\
\hline Multiple territory & $5(1)$ \\
\hline \multicolumn{2}{|l|}{ Endovascular access } \\
\hline \multicolumn{2}{|l|}{ Access site } \\
\hline CFA & $479(100)$ \\
\hline \multicolumn{2}{|l|}{ Side } \\
\hline $\mathrm{Lt}$ & $16(3)$ \\
\hline $\mathrm{Rt}$ & $460(96)$ \\
\hline Both & $3(1)$ \\
\hline
\end{tabular}

$\mathrm{CCA}=$ common carotid artery; ICA = internal carotid artery; $P C A=$ posterior cerebral artery; $\mathrm{VA}=$ vertebral artery.

Values represent the number of patients (\%) unless stated otherwise.

\section{Results}

A total of 479 patients (51\% female) who underwent EVT for ischemic stroke were identified and included in this study. The mean age of this cohort was 71 years, with a standard deviation of 14.5 years and a range of 19-99 years. A descriptive analysis of all 479 patients is shown in Table 1. The most common site of occlusion in our cohort was the middle cerebral artery (MCA; 72\%), followed by the internal carotid artery (15\%) and basilar artery $(12 \%)$. Two patients were found to have occlusions of the posterior cerebral artery, 1 patient had an occlusion of the vertebral artery, and 5 patients were identified with infarcts in multiple territories. One patient had an occlusion of the common carotid artery at the bifurcation with recanalized flow intracranially. Lateralized strokes were slightly more common in the left hemisphere $(47 \%)$ than in the right (41\%), and all patients underwent CFA access, on the right side in $96 \%$ of patients.
TABLE 2. Access-site complications

\begin{tabular}{|c|c|}
\hline Characteristic & Value \\
\hline No. of patients w/ access-site complications & 20 \\
\hline \multicolumn{2}{|l|}{ Sex } \\
\hline M & $12(60)$ \\
\hline $\mathrm{F}$ & $8(40)$ \\
\hline Mean age \pm SD (range), yrs & $64 \pm 19.4(20-95)$ \\
\hline \multicolumn{2}{|l|}{ Endovascular access } \\
\hline \multicolumn{2}{|l|}{ Access site } \\
\hline CFA & $20(100)$ \\
\hline \multicolumn{2}{|l|}{ Side } \\
\hline Lt & $2(10)$ \\
\hline Rt & $18(90)$ \\
\hline Both & $0(0)$ \\
\hline \multicolumn{2}{|l|}{ Access-site complications* } \\
\hline Minor & $8(1.67)$ \\
\hline Groin hematoma & $8(1.67)$ \\
\hline Groin hemorrhage & $0(0)$ \\
\hline Major & $12(2.51)$ \\
\hline Retroperitoneal hematoma & $2(0.42)$ \\
\hline Arteriovenous fistula & $0(0)$ \\
\hline Artery pseudoaneurysm & $4(0.84)$ \\
\hline Artery dissection & $5(1.04)$ \\
\hline Nerve injury & $0(0)$ \\
\hline Arterial occlusion & $1(0.21)$ \\
\hline Wound infection & $0(0)$ \\
\hline
\end{tabular}

All patients presented here experienced only one access-site complication. Values represent the number of patients (\%) unless stated otherwise.

* Percentages are based on the total population of 479 patients.

Primary EVT outcomes from our center have been previously reported and therefore fell outside the scope of this study. ${ }^{18}$ Twenty patients $(4.18 \%)$ in our cohort were identified as having experienced some form of access-site complication (Table 2). Of these patients, the mean age was 64 years and the female-to-male ratio was 2:3. Fourteen patients experienced an access-site complication after vascular closure with an Angio-Seal VCD while the remaining 6 had manual compression (Table 3). Eight (1.67\%) of these patients experienced minor access-site complications, all of which were groin hematomas and none of which were clinically significant (as defined by requiring surgical management or transfusion). One groin hematoma occurred after manual compression and the rest after VCD use. The remaining 12 patients $(2.51 \%)$ experienced arterial dissection $(\mathrm{n}=5,1.04 \%)$, arterial pseudoaneurysm $(\mathrm{n}=4,0.84 \%)$, retroperitoneal hematoma $(\mathrm{n}=2,0.42 \%)$, or arterial occlusion $(\mathrm{n}=1,0.21 \%)$. Dissections were all non-flow-limiting and managed expectantly, except for 1 patient who required surgery to avert leg ischemia. All dissections, after being visualized on femoral artery angiography following EVT, were managed with manual compression. In the single case requiring surgery, an 85-yearold woman with hypertension and known peripheral vascular disease presented with right MCA occlusion and 
TABLE 3. Access-site complications following EVT with VCD and vascular closure via manual compression

\begin{tabular}{lcc}
\hline Access-Site Complication & Angio-Seal VCD & Manual Compression \\
\hline Minor & & \\
\hline$\quad$ Groin hematoma & 7 & 1 \\
\hline Major & 2 & 0 \\
$\quad \begin{array}{l}\text { Retroperitoneal } \\
\text { hematoma }\end{array}$ & 4 & 0 \\
\hline$\quad$ Artery pseudoaneurysm & 0 & 5 \\
\hline Artery dissection & 1 & 0 \\
\hline Arterial occlusion & & \\
\hline
\end{tabular}

underwent attempted EVT. Despite US guidance, a false lumen was created during attempted transfemoral access, and this required surgical repair. Pseudoaneurysms of the CFA, all occurring after VCD deployment, were treated with thrombin injection in 3 patients and managed conservatively in the remaining patient. Neither case of retroperitoneal hematoma, both occurring after VCD, required blood transfusion, and both were managed expectantly. There was a single occurrence of CFA occlusion, attributed to the Angio-Seal device used for access-site closure following successful EVT in a 56-year-old woman, who developed pallor of the right foot. CT angiography demonstrated an occluded CFA with a vertically positioned Angio-Seal footplate, likely caught on the vessel back wall. She underwent successful revascularization with removal of the closure device.

\section{Discussion}

To our knowledge, this constitutes the first retrospective cohort study of US-guided 8F sheath femoral access and is among the largest single-institution observational studies characterizing access-site complications of EVT. Disaggregated, access-site complications in our retrospective cohort treated with EVT using US-guided puncture (4.18\%) were comparable with a 2020 systematic review of 16 randomized controlled trials (RCTs) and 17 observational studies, in which the incidence of complication with no US guidance was reported as 5.13\% and $2.78 \%$, respectively. ${ }^{14}$ Furthermore, our incidence of minor complications $(1.67 \%)$ was markedly lower than rates previously reported in RCTs (4.07\%) and observational studies with or without VCDs $(2.05 \%$ and $2.85 \%$, respectively). However, the incidence of major complications $(2.51 \%)$ in our cohort was greater than those previously reported by Oneissi et al. for these three groups $(0.92 \%, 0.21 \%$, and $0.83 \%$, respectively). ${ }^{14}$ On the other hand, rates of groin pseudoaneurysm and arterial dissection in patients from our cohort were $0.83 \%$ (4/479) and $1.04 \%$ (5/479), respectively, lower than those reported previously. A 2018 review of $8 \mathrm{RCTs}$ reported groin/puncture site hematoma rates of $3.6 \%$, a groin pseudoaneurysm rate of $1 \%$, and an arterial dissection rate of $2 \%,{ }^{21}$ and a 2020 review of 7 prospective trials of EVT with transfemoral access reported a major access-site complication rate of $1.67 \%$ and a total complication rate of $4.59 \% .^{22}$ The latter study also defined major complications as events that resulted in a $>3$-g hemoglobin or a $10 \%$ hematocrit drop, required surgical/radiological intervention, required transfusion, prolonged the patient's stay in the hospital, or resulted in death. ${ }^{22}$

A 2016 retrospective cohort study by Shah and colleagues reported very low rates of access-site complication with large-bore $(8 \mathrm{~F}$ and $9 \mathrm{~F})$ sheaths..$^{23}$ The rate of groin complications $(0.4 \%-0.8 \%)$ reported in their study excluded non-clinically significant hematomas and was considerably lower than other studies have documented. In addition, the authors limited their definition of clinically significant groin complications to groin hematomas, retroperitoneal hematomas, or femoral artery pseudoaneurysms that either required surgical or radiological intervention (including to correct vessel injury due to the deployment of a VCD) or at least one unit of blood transfusion; caused a $>3$-g drop in hemoglobin or $\mathrm{a} \geq 10 \%$ drop in hematocrit; or contributed to the death of a patient. ${ }^{23}$ Although the overall rates of access-site complications were comparatively higher in our cohort, applying these same criteria to our data yielded a similar overall access-site complication rate of $1.0 \%$, as 8 of 8 groin hematomas, 2 of 2 retroperitoneal hematomas, 4 of 5 dissections, and 1 of 4 pseudoaneurysms were managed expectantly (without the requirement of surgical/radiological intervention or blood transfusion).

In addition to the use of US, other factors may also play a role in access-site complications. For example, the concomitant use of anticoagulation or tPA thrombolysis may be associated with increased rates of hemorrhagic accesssite complications due to bleeding diathesis. ${ }^{24-26}$ It has been argued that these patients specifically may benefit the most from the use of VCDs rather than manual compression to decrease these risks. ${ }^{24-26}$ Previous meta-analyses have shown similar rates of access-site complications with the use of VCDs compared with manual compression, as VCDs bear a risk of deployment failure that can lead to groin hematoma, pseudoaneurysm, or even vessel occlusion such as that which occurred in the one patient reported in our cohort. ${ }^{27,28}$ As we utilized VCD for the majority of patients, most access-site complications also occurred after VCD use.

In parallel with advances in transfemoral access for EVT, techniques of securing vascular access through the radial artery have become customary in interventional cardiology and boast lower complication rates than transfemoral approaches. ${ }^{29-31}$ Transradial access is gaining popularity in the neuroendovascular setting as well ${ }^{6,7}$ and its adoption appears to be motivated by apprehension surrounding access-site complications. ${ }^{32}$ Underscoring this, a recent study by Munich and colleagues reported a complication rate of 5.4\% (7/129) with transfemoral access, compared with $0 \%(0 / 44)$ with transradial access, for EVT. ${ }^{8}$ However, the unique challenges presented by transradial access included increased time for vascular access, prevalent radial artery tortuosity, and arterial spasm. ${ }^{32}$ Transfemoral access for EVT therefore continues to play an important role in ischemic stroke management, sustaining the value of US in potentially improving the safety profile of this method.

Interpreting the access-site complication rates in our study population, we propose that the use of US guidance 
was able to further limit the risk of minor access-site complications inherent in EVT. However, this may not extend to increased risk of major access-site complications with the use of larger-bore sheaths. In light of previous studies that identified benefits of US guidance for transfemoral access in patients $>80$ years old or with a BMI of $\geq 30,{ }^{33}$ as well as those with a weak arterial pulse or a leg circumference $>60 \mathrm{~cm},{ }^{34}$ we suggest that the utility of US guidance is likely to be compounded in certain subgroups of patients undergoing EVT. From a technical perspective, US-guided puncture is an elegant replacement for the traditional micropuncture kit, in which a 21-gauge needle is used to perform several exploratory (typically, double-wall) arterial punctures to identify the target site before a sheath is placed. This technique can intuitively be expected to bear greater risk of groin hematoma, although seldom clinically significant, compared with single-wall puncture techniques like that which is enabled by US guidance. Similarly, US guidance is not expected to reduce the rate of certain complications such as retroperitoneal hematoma because the cranial-to-caudal aspect of the femoral head is not visualized. In our cohort, the incidence of major complications may theoretically have been elevated by intentional punctures above the superficial femoral artery bifurcation in order to allow for the Angio-Seal VCD deployment. In addition to decreased rates of transfemoral access complications, US guidance has also been shown to have a superior time-to-access and first-pass success rate. ${ }^{5}$

We identified several limitations of the present study, including a high degree of variability in the literature with respect to reporting access-site complications. Without universal consensus as to what defines a complication, and with limited studies on the incidence of complications, it is challenging to isolate the effect of US guidance on transfemoral access for EVT. While confining our study to a single institution conferred advantages of standardization in procedure and reporting, it also limited the included patients to only those referred to an urban quaternary neurovascular referral center. Additionally, while our data suggest that US guidance can reduce the risks of access-site complications in this setting compared with other published data, this is a retrospective analysis of a cohort of patients who all underwent US-guided puncture, as is standard at our institution. There is no control group of non-US-guided puncture for direct comparison, and our results may not be fully generalizable to other providers who have significant experience in either US-guided or manual puncture. Whether US will confer these same benefits at all centers remains unverified, warranting future investigation with controlled trials, multicenter design, and providers ranging in experience. The present study also offers a univariate analysis of the effect of US guidance on access-site complication rates in EVT, but it must be noted that other variables factor into these rates, including the concomitant use of anticoagulation or tPA thrombolysis, ${ }^{35}$ as well as closure devices.$^{14}$ Finally, the overall rarity of access-site complications in EVT presented obstacles to the robust subgroup analysis of potential complication associations with other factors such as patient age, body habitus, comorbidities, and specific equipment.
EVT continues to develop as the standard of care for patients with acute ischemic stroke, and the refinement of its technique can be expected to benefit thousands of patients per year. Methods to decrease access-site complications may include the increased or routine use of US for transfemoral access, as suggested in our study, the continued development of radial site access techniques, or improvement in VCDs. For transfemoral access, future research directions in ameliorating access-site complication risks in EVT may include multicenter, multivariate analyses of US guidance, anticoagulation, and VCDs in transfemoral puncture.

\section{Conclusions}

Our cohort included a cross section of all patients undergoing EVT at our Canadian center over a period of nearly 7 years, with uniform utilization of a US-guided transfemoral puncture technique. We report decreased rates of minor complications and similar rates of major complications, allowing for differences in reporting criteria, compared with the literature. Our findings suggest that US guidance may be utilized to decrease transfemoral access-site complications for patients undergoing EVT. In addition, while the current practice of EVT contains a high degree of technical variability, we have provided a detailed description of our protocol in an effort to enhance the generalizability of our study results.

\section{References}

1. Saver JL, Goyal M, van der Lugt A, et al. Time to treatment with endovascular thrombectomy and outcomes from ischemic stroke: a meta-analysis. JAMA. 2016;316(12):12791288.

2. Mokin M, Ansari SA, McTaggart RA, et al. Indications for thrombectomy in acute ischemic stroke from emergent large vessel occlusion (ELVO): report of the SNIS Standards and Guidelines Committee. J Neurointerv Surg. 2019;11(3): 215-220.

3. Hassan AE, Chaudhry SA, Grigoryan M, et al. National trends in utilization and outcomes of endovascular treatment of acute ischemic stroke patients in the mechanical thrombectomy era. Stroke. 2012;43(11):3012-3017.

4. Mehta T, Male S, Quinn C, et al. Institutional and provider variations for mechanical thrombectomy in the treatment of acute ischemic stroke: a survey analysis. J Neurointerv Surg. 2019;11(9):884-890.

5. Sorenson TJ, Nicholson PJ, Hilditch CA, et al. A lesson from cardiology: the argument for ultrasound-guided femoral artery access in interventional neuroradiology. World Neurosurg. 2019;126:124-128.

6. Khanna O, Sweid A, Mouchtouris N, et al. Radial artery catheterization for neuroendovascular procedures. Stroke. 2019;50(9):2587-2590.

7. Kühn AL, de Macedo Rodrigues K, Singh J, et al. Distal radial access in the anatomical snuffbox for neurointerventions: a feasibility, safety, and proof-of-concept study. $\mathrm{J} \mathrm{Neu-}$ rointerv Surg. 2020;12(8):798-801.

8. Munich SA, Vakharia K, McPheeters MJ, et al. Transition to transradial access for mechanical thrombectomy-lessons learned and comparison to transfemoral access in a singlecenter case series. Oper Neurosurg (Hagerstown). 2020; 19(6):701-707.

9. Cord BJ, Kodali S, Strander S, et al. Direct carotid puncture for mechanical thrombectomy in acute ischemic stroke pa- 
tients with prohibitive vascular access. J Neurosurg. 2021; 135(1):53-63.

10. Miszczuk M, Bauknecht HC, Kleine JF, et al. Direct puncture of the carotid artery as a bailout vascular access technique for mechanical thrombectomy in acute ischemic stroke - the revival of an old technique in a modern setting. Neuroradiology. 2021;63(2):275-283.

11. Semeraro V, Gasparrini F, Vidali S, Gandini R. Direct ultrasound-guided puncture of vertebral artery V2 segment during mechanical thrombectomy. BMJ Case Rep. 2021;14(1): e238979.

12. Desai JA, Almekhlafi MA, Hill MD, et al. Ultrasound guided V3 segment vertebral artery direct percutaneous puncture for basilar artery mechanical thrombectomy in acute stroke: a technical report. BMJ Case Rep. 2013;2013:bcr2012010601.

13. Ortiz D, Jahangir A, Singh M, et al. Access site complications after peripheral vascular interventions: incidence, predictors, and outcomes. Circ Cardiovasc Interv. 2014;7(6): 821-828.

14. Oneissi M, Sweid A, Tjoumakaris S, et al. Access-site complications in transfemoral neuroendovascular procedures: a systematic review of incidence rates and management strategies. Oper Neurosurg (Hagerstown). 2020;19(4):353-363.

15. Daou BJ, Pandey AS. Commentary: access-site complications in transfemoral neuroendovascular procedures: a systematic review of incidence rates and management strategies. Oper Neurosurg (Hagerstown). 2020;19(4):E335-E336.

16. Pitta SR, Prasad A, Kumar G, et al. Location of femoral artery access and correlation with vascular complications. Catheter Cardiovasc Interv. 2011;78(2):294-299.

17. Kwok CS, Kontopantelis E, Kinnaird T, et al. Retroperitoneal hemorrhage after percutaneous coronary intervention: incidence, determinants, and outcomes as recorded by the British Cardiovascular Intervention Society. Circ Cardiovasc Interv. 2018;11(2):e005866.

18. Sarzetto F, Gupta S, Alotaibi NM, et al. Outcome evaluation of acute ischemic stroke patients treated with endovascular thrombectomy: a single-institution experience in the era of randomized controlled trials. World Neurosurg. 2017;99: 593-598.

19. Pasarikovski CR, Tanner BK, Marques AJP, et al. Neurointerventional procedural complications in a growing Canadian regional stroke center: single hospital experience analysis in the context of recommended case volumes. World Neurosurg. 2019;127:e94-e100.

20. von Elm E, Altman DG, Egger M, et al. The Strengthening the Reporting of Observational Studies in Epidemiology (STROBE) statement: guidelines for reporting observational studies. Ann Intern Med. 2007;147(8):573-577.

21. Balami JS, White PM, McMeekin PJ, et al. Complications of endovascular treatment for acute ischemic stroke: Prevention and management. Int J Stroke. 2018;13(4):348-361.

22. Shapiro SZ, Sabacinski KA, Mantripragada K, et al. Accesssite complications in mechanical thrombectomy for acute ischemic stroke: a review of prospective trials. AJNR Am J Neuroradiol. 2020;41(3):477-481.

23. Shah VA, Martin CO, Hawkins AM, et al. Groin complications in endovascular mechanical thrombectomy for acute ischemic stroke: a 10-year single center experience. J Neurointerv Surg. 2016;8(6):568-570.

24. Patil MS, Jayaraman MV, Ahn SH. Vascular closure devices in stroke patients receiving tissue plasminogen activator: a retrospective analysis from an academic tertiary medical center and a teaching community hospital stroke database. Clin Neurol Neurosurg. 2017;157:22-24.
25. Wilensky JA, Ali AT, Moursi MM, et al. Outcomes after arterial endovascular procedures performed in patients with an elevated international normalized ratio. Ann Vasc Surg. 2015; 29(1):22-27.

26. Naddaf A, Williams S, Hasanadka R, et al. Predictors of groin access pseudoaneurysm complication: a 10-year institutional experience. Vasc Endovascular Surg. 2020;54(1): $42-46$.

27. Rao SV, Stone GW. Arterial access and arteriotomy site closure devices. Nat Rev Cardiol. 2016;13(11):641-650.

28. Jiang J, Zou J, Ma H, et al. Network meta-analysis of randomized trials on the safety of vascular closure devices for femoral arterial puncture site haemostasis. Sci Rep. 2015;5: 13761.

29. Jolly SS, Amlani S, Hamon M, et al. Radial versus femoral access for coronary angiography or intervention and the impact on major bleeding and ischemic events: a systematic review and meta-analysis of randomized trials. Am Heart $J$. 2009;157(1):132-140.

30. Ferrante G, Rao SV, Jüni P, et al. Radial versus femoral access for coronary interventions across the entire spectrum of patients with coronary artery disease: a meta-analysis of randomized trials. JACC Cardiovasc Interv. 2016;9(14): 1419-1434.

31. Jolly SS, Yusuf S, Cairns J, et al. Radial versus femoral access for coronary angiography and intervention in patients with acute coronary syndromes (RIVAL): a randomised, parallel group, multicentre trial. Lancet. 2011;377(9775): 1409-1420.

32. Das S, Ramesh S, Velagapudi L, et al. Adoption of the transradial approach for neurointerventions: a national survey of current practitioners. J Stroke Cerebrovasc Dis. 2021;30(3): 105589.

33. Kalish J, Eslami M, Gillespie D, et al. Routine use of ultrasound guidance in femoral arterial access for peripheral vascular intervention decreases groin hematoma rates. $J$ Vasc Surg. 2015;61(5):1231-1238.

34. Dudeck O, Teichgraeber U, Podrabsky P, et al. A randomized trial assessing the value of ultrasound-guided puncture of the femoral artery for interventional investigations. Int J Cardiovasc Imaging. 2004;20(5):363-368.

35. Saver JL, Adeoye O. Intravenous thrombolysis before endovascular thrombectomy for acute ischemic stroke. JAMA. 2021;325(3):229-231.

\section{Disclosures}

The authors report no conflict of interest concerning the materials or methods used in this study or the findings specified in this paper.

\section{Author Contributions}

Conception and design: Yang, Brenna. Acquisition of data: Brenna, Ku, Pasarikovski. Analysis and interpretation of data: Brenna. Drafting the article: Brenna. Critically revising the article: Yang, Brenna, Ku. Reviewed submitted version of manuscript: Yang, Priola, Dyer, Howard, Kumar, da Costa. Statistical analysis: Brenna. Administrative/technical/material support: Yang, Ku, Pasarikovski, Priola, Dyer, Howard, Kumar, da Costa. Study supervision: Yang.

\section{Correspondence}

Victor X. D. Yang: Sunnybrook Health Sciences Centre, Toronto, ON, Canada. victor.yang@sunnybrook.ca. 